\title{
Correction to: Efficacy of radiofrequency ablation in autonomous functioning thyroid nodules. A systematic review and meta-analysis
}

\author{
Roberto Cesareo ${ }^{1}$. Andrea Palermo ${ }^{2}$. Domenico Benvenuto ${ }^{3}$ - Eleonora Cella ${ }^{3}$. Valerio Pasqualini ${ }^{4}$. \\ Stella Bernardi ${ }^{5} \cdot$ Fulvio Stacul $^{6}$ - Silvia Angeletti ${ }^{7} \cdot$ Giovanni Mauri $^{8} \cdot$ Massimo Ciccozzi $^{3} \cdot$ Pierpaolo Trimboli $^{9}$
}

Published online: 26 April 2019

(C) Springer Science+Business Media, LLC, part of Springer Nature 2019

\section{Correction to: Reviews in Endocrine and Metabolic Disorders https://doi.org/10.1007/s11154-019-09487-y}

The authors of this paper declare that their correct family and first names and their correct affiliations are shown in this correction paper.

The original article has been corrected.

Pierpaolo Trimboli

pierpaolo.trimboli@eoc.ch

1 Thyroid and Metabolic Bone Diseases Center, Santa Maria Goretti Hospital, Latina, Italy

2 Department of Endocrinology, University Campus Biomedico, Rome, Italy

3 Unit of Medical statistic and Molecular Epidemiology, University Campus Bio-Medico, Rome, Italy

4 Departments of Radiology, Santa Maria Goretti Hospital, Latina, Italy
Department of Medical Sciences, University of Trieste, Cattinara Teaching Hospital, Trieste, Italy

6 Radiology Department, Maggiore Teaching Hospital, ASUITS, Trieste, Italy

7 UOC Laboratory Medicine, University Hospital Campus Bio-Medico of Rome, Rome, Italy

8 Division of Interventional Radiology, European Institute of Oncology, IRCCS, Milan, Italy

9 Department of Nuclear Medicine and Thyroid Centre, Oncology Institute of Southern Switzerland, Bellinzona, Switzerland 